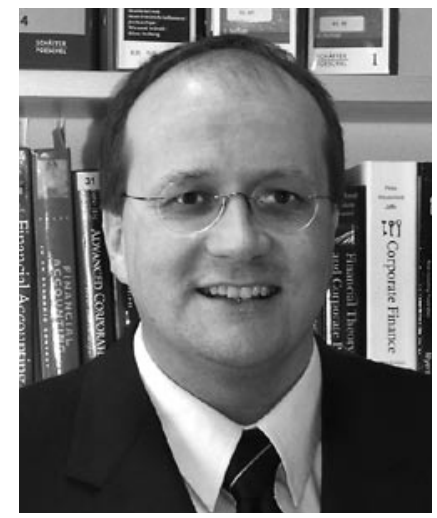

Dirk Hachmeister

\section{Controlling \\ und Liquidität}

\author{
Liebe Leser,
}

als wir die Themen der Zeitschrift für Controlling \& Management ursprünglich planten, wollten wir Sie über die Ideen zur Gestaltung der Passivseite und insbesondere der Minimierung der Fremd- und Eigenkapitalkosten informieren. Aus theoretischer Sicht ist diese Fragestellung sicherlich zeitlos, aus Sicht der Praxis - mit Blick auf die Finanz- und Wirtschaftskrise - hat sich jedoch die Priorität stark verändert: Ging es bis letztes Jahr oft um eine Optimierung der Finanzierung - vielleicht auch mit neuen, innovativen Produkten - steht im Augenblick vielmehr die Sicherstellung der Finanzierung, insbesondere der Liquidität im Fokus. Es gilt wieder:"Cash is King“. Aber auch das Thema der Insolvenz hat heute einen anderen Stellenwert. Diesen veränderten Anforderungen haben wir uns bei der Gestaltung des Hefts gestellt und bei der Auswahl der Artikel beachtet.

So steht in den beiden Themenbeiträgen von Patrick Buchmann und Dr. Carsten Kruppe die Finanzkrise und ihre Herausforderungen im Mittelpunkt des Interesses: Herr Buchmann untersucht in seinem Beitrag wie Liquiditätsengpässe durch die Optimierung von Vorräten, Forderungen und Verbindlichkeiten vermieden werden können. Anhand praktischer Ansatzpunkte erläutert er die spezifischen Anforderungen und Erfolgsfaktoren des Working Capital Management in der Krise. Herr Dr. Kruppe zeigt am Beispiel einer Großinvestition auf, welche Bedeutung eine zuverlässige Finanzplanung für die Planung und Steuerung der Fremdfinanzierung hat.

Im Transferbeitrag von Frank Bockisch und mir wird auf einen modelltheoretischen Beitrag verwiesen, der sich mit dem Verlauf von Insolvenzverfahren beschäftigt. Der Autor stellt die These auf, dass das Insolvenzverfahren einen notwendigen Lern- und Selektionsmechanismus verkörpert, der es den Gläubigern überhaupt erst ermöglicht, wirtschaftlich starke von wirtschaftlich schwachen Unternehmen zu unterscheiden - und entsprechend eine effiziente Liquidationsentscheidung zu treffen. In einem empirischen Beitrag wird die Bedeutung verschiedener nationaler Insolvenzordnungen im Rahmen von Restrukturierungsentscheidungen untersucht. Dabei wird auch deutlich, dass Banken aufgrund national unterschiedlicher Gläubigerrechte ihre Kreditvergabepraxis sowie ihr Vorgehen bei Restrukturierungen entsprechend unterschiedlich ausgestalten.

Auch wenn die Themen der beiden Transferbeiträge sicherlich eher unerfreulich sind und bei der ursprünglichen Themenplanung nicht auf der Agenda standen, sind wir der Meinung, dass die Ideen und Erkenntnisse einem breiteren Publikum zugänglich gemacht werden sollten. Das Thema ist viel zu relevant, als dass sich nur Spezialisten mit dieser Problematik auseinandersetzen. Versteht man Controlling als Koordination von Führungsfunktionen, so kann die Abstimmung von Informationsbeschaffung - auch im Rahmen einer Insolvenzentscheidung - als wichtige Aufgabe des Controllers bezeichnet werden.

Wir hoffen, dass es uns gelungen ist, Sie auch in turbulenten Zeiten mit Anregungen versorgen zu können.

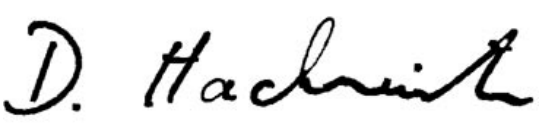

Ihr Dirk Hachmeister 